\title{
Generalized difference strongly summable sequence spaces of fuzzy real numbers defined by ideal convergence and Orlicz function
}

\section{Adem Kılıçman ${ }^{1}$ and Stuti Borgohain 2*}

\section{"Correspondence:}

stutiborgohain@yahoo.com

${ }^{2}$ Department of Mathematics,

Indian Institute of Technology,

Bombay Powai: 400076, Mumbai,

Maharashtra, India

Full list of author information is

available at the end of the article

\begin{abstract}
We study some new generalized difference strongly summable sequence spaces of fuzzy real numbers using ideal convergence and an Orlicz function in connection with de la Vallèe Poussin mean. We give some relations related to these sequence spaces also.

MSC: 40A05; 40A25; 40A30; 40C05
\end{abstract}

Keywords: Orlicz function; difference operator; ideal convergence; de la Vallèe Poussin mean

\section{Introduction}

Let $\ell_{\infty}, c$ and $c_{0}$ be the Banach space of bounded, convergent and null sequences $x=\left(x_{k}\right)$, respectively, with the usual norm $\|x\|=\sup _{n}\left|x_{n}\right|$.

A sequence $x \in \ell_{\infty}$ is said to be almost convergent if all of its Banach limits coincide.

Let $\hat{c}$ denote the space of all almost convergent sequences.

Lorentz [1] proved that,

$$
\hat{c}=\left\{x \in \ell_{\infty}: \lim _{m} t_{m, n}(x) \text { exists uniformly in } n\right\},
$$

where

$$
t_{m, n}(x)=\frac{x_{n}+x_{n+1}+\cdots+x_{m+n}}{m+1} .
$$

The following space of strongly almost convergent sequence was introduced by Mad$\operatorname{dox}[2]$ :

$$
[\hat{c}]=\left\{x \in \ell_{\infty}: \lim _{m} t_{m, n}(|x-L e|) \text { exists uniformly in } n \text { for some } L\right\},
$$

where, $e=(1,1, \ldots)$.

Let $\sigma$ be a one-to-one mapping from the set of positive integers into itself such that $\sigma^{m}(n)=\sigma^{m-1}(\sigma(n)), m=1,2,3, \ldots$, where $\sigma^{m}(n)$ denotes the $m$ th iterative of the mapping $\sigma$ in $n$, see [3].

○2013 Kliçman and Borgohain; licensee Springer. This is an Open Access article distributed under the terms of the Creative Commons Attribution License (http://creativecommons.org/licenses/by/2.0), which permits unrestricted use, distribution, and reproduction in any medium, provided the original work is properly cited. 
Schaefer [3] proved that

$$
V_{\sigma}=\left\{x \in \ell_{\infty}: \lim _{k} t_{k m}(x)=L \text { uniformly in } m \text { for some } L=\sigma-\lim x\right\},
$$

where,

$$
t_{k m}(x)=\frac{x+x_{\sigma(m)}+\cdots+x_{\sigma^{k}(m)}}{k+1}, \quad t_{-1, m}=0 .
$$

Thus, we say that a bounded sequence $x=\left(x_{k}\right)$ is $\sigma$-convergent if and only if $x \in V_{\sigma}$ such that $\sigma^{k}(n) \neq n$ for all $n \geq 0, k \geq 1$.

A sequence $x=\left(x_{k}\right)$ is said to be strongly $\sigma$-convergent (Mursaleen [4]) if there exists a number $\ell$ such that

$$
\frac{1}{k} \sum_{i=1}^{k}\left|x_{\sigma^{i}(m)}-\ell\right| \rightarrow 0, \quad \text { as } k \rightarrow \infty \text { uniformly in } m .
$$

We write $\left[V_{\sigma}\right]$ to denote the set of all strong $\sigma$-convergent sequences, and when (1) holds, we write $\left[V_{\sigma}\right]-\lim x=\ell$.

Taking $\sigma(m)=m+1$, we obtain $\left[V_{\sigma}\right]=[\hat{c}]$. Then the strong $\sigma$-convergence generalizes the concept of strong almost convergence.

We also note that

$$
\left[V_{\sigma}\right] \subset V_{\sigma} \subset \ell_{\infty}
$$

The notion of ideal convergence was first introduced by Kostyrko et al. [5] as a generalization of statistical convergence, which was later studied by many other authors.

An Orlicz function is a function $M:[0, \infty) \rightarrow[0, \infty)$, which is continuous, nondecreasing and convex with $M(0)=0, M(x)>0$, for $x>0$ and $M(x) \rightarrow \infty$, as $x \rightarrow \infty$.

Lindenstrauss and Tzafriri [6] used the idea of Orlicz function to construct the sequence space,

$$
\ell_{M}=\left\{\left(x_{k}\right) \in w: \sum_{k=1}^{\infty} M\left(\frac{|x|}{\rho}\right)<\infty, \text { for some } \rho>0\right\} .
$$

The space $\ell_{M}$ with the norm

$$
\|x\|=\inf \left\{\rho>0: \sum_{k=1}^{\infty} M\left(\frac{\left|x_{k}\right|}{\rho}\right) \leq 1\right\}
$$

becomes a Banach space, which is called an Orlicz sequence space.

Kizmaz [7] studied the difference sequence spaces $\ell_{\infty}(\Delta), c(\Delta)$ and $c_{0}(\Delta)$ of crisp sets. The notion is defined as follows:

$$
Z(\Delta)=\left\{x=\left(x_{k}\right):\left(\Delta x_{k}\right) \in Z\right\}
$$

for $Z=\ell_{\infty}, c$ and $c_{0}$, where $\Delta x=\left(\Delta x_{k}\right)=\left(x_{k}-x_{k+1}\right)$, for all $k \in N$. 
The spaces above are Banach spaces, normed by

$$
\|x\|_{\Delta}=\left|x_{1}\right|+\sup _{k}\left|\Delta x_{k}\right| .
$$

The generalized difference is defined as follows:

For $m \geq 1$ and $n \geq 1$,

$$
Z\left(\Delta_{m}^{n}\right)=\left\{x=\left(x_{k}\right):\left(\Delta_{m}^{n} x_{k}\right) \in Z\right\}
$$

for $Z=\ell_{\infty}, c$ and $c_{0}$.

This generalized difference has the following binomial representation:

$$
\Delta_{m}^{n} x_{k}=\sum_{r=0}^{n}(-1)^{r}\left(\begin{array}{l}
n \\
r
\end{array}\right) x_{k+r m}
$$

The concept of fuzzy set theory was introduced by Zadeh in the year 1965. It has been applied for the studies in almost all the branches of science, where mathematics is used. Workers on sequence spaces have also applied the notion and introduced sequences of fuzzy real numbers and studied their different properties.

\section{Definitions and preliminaries}

A fuzzy real number $X$ is a fuzzy set on $R$, i.e., a mapping $X: R \rightarrow I(=[0,1])$ associating each real number $t$ with its grade of membership $X(t)$.

A fuzzy real number $X$ is called convex if $X(t) \geq X(s) \wedge X(r)=\min (X(s), X(r))$, where $s<t<r$.

If there exists $t_{0} \in R$ such that $X\left(t_{0}\right)=1$, then the fuzzy real number $X$ is called normal. A fuzzy real number $X$ is said to be upper semicontinuous if for each $\varepsilon>0, X^{-1}([0, a+\varepsilon))$, for all $a \in I$, it is open in the usual topology of $R$.

The class of all upper semicontinuous, normal, convex fuzzy real numbers is denoted by $R(I)$.

Define $\bar{d}: R(I) \times R(I) \rightarrow R$ by $\bar{d}(X, Y)=\sup _{0<\alpha \leq 1} d\left(X^{\alpha}, Y^{\alpha}\right)$, for $X, Y \in R(I)$. Then it is well known that $(R(I), \bar{d})$ is a complete metric space.

A sequence $X=\left(X_{n}\right)$ of fuzzy real numbers is said to converge to the fuzzy number $X_{0}$, if for every $\varepsilon>0$, there exists $n_{0} \in N$ such that $\bar{d}\left(X_{n}, X_{0}\right)<\varepsilon$ for all $n \geq n_{0}$.

Let $X$ be a nonempty set. Then a family of sets $I \subseteq 2^{X}$ (power sets of $X$ ) is said to be an ideal if $I$ is additive, i.e., $A, B \in I \Rightarrow A \cup B \in I$ and hereditary, i.e., $A \in I, B \subseteq A \Rightarrow B \in I$.

A sequence $\left(X_{k}\right)$ of fuzzy real numbers is said to be $I$-convergent to a fuzzy real number $X_{0} \in X$ if for each $\varepsilon>0$, the set

$$
E(\varepsilon)=\left\{k \in N: \bar{d}\left(X_{k}, X_{0}\right) \geq \varepsilon\right\} \text { belongs to } I .
$$

The fuzzy number $X_{0}$ is called the $I$-limit of the sequence $\left(X_{k}\right)$ of fuzzy numbers, and we write $I-\lim X_{k}=X_{0}$.

The generalized de la Vallé-Poussin mean is defined by

$$
t_{n}(x)=\frac{1}{\lambda_{n}} \sum_{k \in I_{n}} x_{k},
$$

where $I_{n}=\left[n-\lambda_{n}+1, n\right]$ for $n=1,2, \ldots$ 
Then a sequence $x=\left(x_{k}\right)$ is said to be $(V, \lambda)$-summable to a number $L[8]$ if $t_{n}(x) \rightarrow L$ as $n \rightarrow \infty$, and we write

$$
\begin{aligned}
& {[V, \lambda]_{0}=\left\{x: \lim _{n} \frac{1}{\lambda_{n}} \sum_{k \in I_{n}}\left|x_{k}\right|=0\right\},} \\
& {[V, \lambda]=\left\{x: x-\ell e \in[V, \lambda]_{0} \text { for some } \ell \in C\right\},} \\
& {[V, \lambda]_{\infty}=\left\{x: \sup _{n} \frac{1}{\lambda_{n}} \sum_{k \in I_{n}}\left|x_{k}\right|<\infty\right\}}
\end{aligned}
$$

for the sets of sequences that are, respectively, strongly summable to zero, strongly summable, and strongly bounded by de la Vallé-Poussin method.

We also note that Nuray and Savas [9] defined the sets of sequence spaces such as strongly $\sigma$-summable to zero, strongly $\sigma$-summable and strongly $\sigma$-bounded with respect to the modulus function, see [10].

In this article, we define some new sequence spaces of fuzzy real numbers by using Orlicz function with the notion of generalized de la Vallèe Poussin mean, generalized difference sequences and ideals. We will also introduce and examine certain new sequence spaces using the tools above.

\section{Main results}

Let $I$ be an admissible ideal of $N$, let $M$ be an Orlicz function. Let $r=\left(r_{k}\right)$ be a sequence of real numbers such that $r_{k}>0$ for all $k$, and $\sup _{k} r_{k}<\infty$. This assumption is made throughout the paper.

In this article, we have introduced the following sequence spaces,

$$
\begin{aligned}
& {\left[V_{\sigma}, \lambda, \Delta_{p}^{q}, M, r\right]_{0}^{I(F)}} \\
& =\left\{\left(X_{k}\right) \in w^{F}: \forall \varepsilon>0\left\{n \in N: \lim _{n} \frac{1}{\lambda_{n}} \sum_{k \in I_{n}}\left\{M\left(\frac{\bar{d}\left(\Delta_{p}^{q} X_{\sigma^{k}(m)}, \overline{0}\right)}{\rho}\right)\right\}^{r_{k}} \geq \varepsilon,\right.\right. \\
& \quad \text { uniformly in } m\} \in I\} \text { for some } \rho>0, \\
& {\left[V_{\sigma}, \lambda, \Delta_{p}^{q}, M, r\right]^{I(F)}} \\
& =\left\{\left(X_{k}\right) \in w^{F}: \forall \varepsilon>0\left\{n \in N: \lim _{n} \frac{1}{\lambda_{n}} \sum_{k \in I_{n}}\left\{M\left(\frac{\bar{d}\left(\Delta_{p}^{q} X_{\sigma^{k}(m)}, X_{0}\right)}{\rho}\right)\right\}^{r_{k}} \geq \varepsilon\right\} \in I\right\}
\end{aligned}
$$

for some $\rho>0, X_{0} \in R(I)$,

$$
\begin{aligned}
& {\left[V_{\sigma}, \lambda, \Delta_{p}^{q}, M, r\right]_{\infty}^{I(F)}} \\
& \quad=\left\{\left(X_{k}\right) \in w^{F}: \exists K>0 \text {, s.t. }\left\{\sup _{n, m} \frac{1}{\lambda_{n}} \sum_{k \in I_{n}}\left\{M\left(\frac{\bar{d}\left(\Delta_{p}^{q} X_{\sigma^{k}(m)}, \overline{0}\right.}{\rho}\right)\right\}^{r_{k}} \geq K\right\} \in I\right\}
\end{aligned}
$$

for some $\rho>0$. 
In particular, if we take $r_{k}=1$ for all $k$, we have

$$
\begin{aligned}
& {\left[V_{\sigma}, \lambda, \Delta_{p}^{q}, M\right]_{0}^{I(F)}} \\
& =\left\{\left(X_{k}\right) \in w^{F}: \forall \varepsilon>0\left\{n \in N: \lim _{n} \frac{1}{\lambda_{n}} \sum_{k \in I_{n}}\left\{M\left(\frac{\bar{d}\left(\Delta_{p}^{q} X_{\sigma^{k}(m)} \overline{0}\right)}{\rho}\right)\right\} \geq \varepsilon,\right.\right. \\
& \quad \text { uniformly in } m\} \in I\} \text { for some } \rho>0, \\
& {\left[V_{\sigma}, \lambda, \Delta_{p}^{q}, M\right]^{I(F)}} \\
& =\left\{\left(X_{K}\right) \in w^{F}: \forall \varepsilon>0\left\{n \in N: \lim _{n} \frac{1}{\lambda_{n}} \sum_{k \in I_{n}}\left\{M\left(\frac{\bar{d}\left(\Delta_{p}^{q} X_{\sigma^{k}(m)}, X_{0}\right)}{\rho}\right)\right\} \geq \varepsilon\right\} \in I\right\}
\end{aligned}
$$

for some $\rho>0, X_{0} \in R(I)$,

$$
\begin{aligned}
& {\left[V_{\sigma}, \lambda, \Delta_{p}^{q}, M\right]_{\infty}^{I(F)}} \\
& \quad=\left\{\left(X_{k}\right) \in w^{F}: \exists K>0\left\{\sup _{n, m} \frac{1}{\lambda_{n}} \sum_{k \in I_{n}}\left\{M\left(\frac{\bar{d}\left(\Delta_{p}^{q} X_{\sigma^{k}(m)}, \overline{0}\right)}{\rho}\right)\right\} \geq K\right\} \in I\right\} .
\end{aligned}
$$

Similarly, when $\sigma(m)=m+1$, then $\left[V, \lambda, \Delta_{p}^{q}, M, r\right]_{0}^{I(F)},\left[V_{\sigma}, \lambda, \Delta_{p}^{q}, M, r\right]^{I(F)}$ and $\left[V_{\sigma}, \lambda, \Delta_{p}^{q}\right.$, $M, r]_{\infty}^{I(F)}$ are reduced to

$$
\begin{aligned}
& {\left[\hat{V}, \lambda, \Delta_{p}^{q}, M, r\right]_{0}^{I(F)}} \\
& \quad=\left\{\left(X_{k}\right) \in w^{F}: \forall \varepsilon>0\left\{n \in N: \lim _{n} \frac{1}{\lambda_{n}} \sum_{k \in I_{n}}\left\{M\left(\frac{\bar{d}\left(\Delta_{p}^{q} X_{k+m}, \overline{0}\right)}{\rho}\right)\right\}^{r_{k}} \geq \varepsilon\right\} \in I\right\}
\end{aligned}
$$

uniformly in $m$ for some $\rho>0$.

$$
\begin{aligned}
& {\left[\hat{V}, \lambda, \Delta_{p}^{q}, M, r\right]^{I(F)}} \\
& \quad=\left\{\left(X_{k}\right) \in w^{F}: \forall \varepsilon>0\left\{n \in N: \lim _{n} \frac{1}{\lambda_{n}} \sum_{k \in I_{n}}\left\{M\left(\frac{\bar{d}\left(\Delta_{p}^{q} X_{k+m}, X_{0}\right)}{\rho}\right)\right\}^{r_{k}} \geq \varepsilon\right\} \in I\right\}
\end{aligned}
$$

for some $\rho>0, X_{0} \in R(I)$.

$$
\left[\hat{V}, \lambda, \Delta_{p}^{q}, M, r\right]_{\infty}^{I(F)}=\left\{x: \exists K>0 \text {, s.t. }\left\{\sup _{n, m} \frac{1}{\lambda_{n}} \sum_{k \in I_{n}}\left\{M\left(\frac{\bar{d}\left(\Delta_{p}^{q} X_{k+m}, \overline{0}\right.}{\rho}\right)\right\}^{r_{k}} \geq k\right\} \in I\right\}
$$

for some $\rho>0$.

In particular, if we put $r_{k}=r$, for all $k$, then we have the spaces

$$
\begin{aligned}
& {\left[\hat{V}, \lambda, \Delta_{p}^{q}, M, r\right]_{0}^{I(F)}=\left[\hat{V}, \lambda, \Delta_{p}^{q}, M\right]_{0}^{I(F)},} \\
& {\left[\hat{V}, \lambda, \Delta_{p}^{q}, M, r\right]^{I(F)}=\left[\hat{V}, \lambda, \Delta_{p}^{q}, M\right]^{I(F)},} \\
& {\left[\hat{V}, \lambda, \Delta_{p}^{q}, M, r\right]_{\infty}^{I(F)}=\left[\hat{V}, \lambda, \Delta_{p}^{q}, M\right]_{\infty}^{I(F)} .}
\end{aligned}
$$

Further, when $\lambda_{n}=n$, for $n=1,2, \ldots$, the sets $\left[\hat{V}, \lambda, \Delta_{p}^{q}, M\right]_{0}^{I(F)}$ and $\left[\hat{V}, \lambda, \Delta_{p}^{q}, M\right]^{I(F)}$ are reduced to $\left[\hat{c}_{0}\left(M, \Delta_{p}^{q}\right)\right]^{I(F)}$ and $\left[\hat{c}\left(M, \Delta_{p}^{q}\right)\right]^{I(F)}$, respectively. 
Now, if we consider $M(x)=x$, then we can easily obtain

$$
\begin{aligned}
& {\left[V_{\sigma}, \lambda, \Delta_{p}^{q}, r\right]_{0}^{I(F)}} \\
& =\left\{\left(X_{k}\right) \in w^{F}: \forall \varepsilon>0\left\{n \in N: \lim _{n} \frac{1}{\lambda_{n}} \sum_{k \in I_{n}}\left(\bar{d}\left(\Delta_{p}^{q} X_{\sigma^{k}(m)}, \overline{0}\right)\right)^{r_{k}} \geq \varepsilon,\right.\right. \\
& \quad \text { uniformly in } m\} \in I\}, \\
& {\left[V_{\sigma}, \lambda, \Delta_{p}^{q}, r\right]^{I(F)}} \\
& \quad=\left\{\left(X_{k}\right) \in w^{F}: \forall \varepsilon>0\left\{n \in N: \lim _{n} \frac{1}{\lambda_{n}} \sum_{k \in I_{n}}\left(\bar{d}\left(\Delta_{p}^{q} X_{\sigma^{k}(m)}, X_{0}\right)\right)^{r_{k}} \geq \varepsilon\right\} \in I\right\}
\end{aligned}
$$

for $X_{0} \in R(I)$.

$$
\left[V_{\sigma}, \lambda, \Delta_{p}^{q}, r\right]_{\infty}^{I(F)}=\left\{\left(X_{k}\right) \in w^{F}: \exists K>0 \text { s.t. }\left\{\sup _{n, m} \frac{1}{\lambda_{n}} \sum_{k \in I_{n}}\left(\bar{d}\left(\Delta_{p}^{q} X_{\sigma^{k}(m)}, \overline{0}\right)\right)^{r_{k}} \geq K\right\} \in I\right\} .
$$

If $X \in\left[V_{\sigma}, \lambda, \Delta_{p}^{q}, M, r\right]^{I(F)}$ with $\left\{\frac{1}{\lambda_{n}} \sum_{k \in I_{n}}\left\{M\left(\frac{\bar{d}\left(\Delta_{p}^{q} X_{\sigma^{k}(m)}, X_{0}\right)}{\rho}\right)\right\}^{r_{k}} \geq \varepsilon\right\} \in I$ as $n \rightarrow \infty$ uniformly in $m$, then we write $X_{k} \rightarrow X_{0} \in\left[V_{\sigma}, \lambda, \Delta_{p}^{q}, M, r\right]^{I(F)}$.

The following well-known inequality will be used later.

$$
\text { If } 0 \leq r_{k} \leq \sup r_{k}=H \text { and } C=\max \left(1,2^{H-1}\right) \text {, then }
$$

$$
\left|a_{k}+b_{k}\right|^{r_{k}} \leq C\left\{\left|a_{k}\right|^{r_{k}}+\left|b_{k}\right|^{r_{k}}\right\}
$$

for all $k$ and $a_{k}, b_{k} \in C$.

Lemma 3.1 (See [9]) Let $r_{k}>0, s_{k}>0$. Then $c_{0}(s) \subset c_{0}(r)$ if and only if $\lim _{k \rightarrow \infty}$ inf $\frac{r_{k}}{s_{k}}>0$, where $c_{0}(r)=\left\{x:\left|x_{k}\right|^{r_{k}} \rightarrow 0\right.$ as $\left.k \rightarrow \infty\right\}$.

Note that no other relation between $\left(r_{k}\right)$ and $\left(s_{k}\right)$ is needed in Lemma 3.1.

Theorem 3.2 Let $\lim _{k \rightarrow \infty} \inf r_{k}>0$. Then $X_{k} \rightarrow X_{0}$ implies that $X_{k} \rightarrow X_{0} \in\left[V_{\sigma}, \lambda, \Delta_{p}^{q}\right.$, $M, r]^{I(F)}$. Let $\lim _{k \rightarrow \infty} r_{k}=r>0$. If $X_{k} \rightarrow X_{0} \in\left[V_{\sigma}, \lambda, \Delta_{p}^{q}, M, r\right]^{I(F)}$, then $X_{0}$ is unique.

Proof Let $X_{k} \rightarrow X_{0}$.

By the definition of Orlicz function, we have for all $\varepsilon>0$,

$$
\left\{n \in N: \lim _{n} \frac{1}{\lambda_{n}} \sum_{k \in I_{n}} M\left(\frac{\bar{d}\left(\Delta_{p}^{q} X_{\sigma^{k}(m)}, X_{0}\right)}{\rho}\right) \geq \varepsilon\right\} \in I .
$$

Since $\lim _{k \rightarrow \infty} \inf r_{k}>0$, it follows that

$$
\left\{n \in N: \lim _{n} \frac{1}{\lambda_{n}} \sum_{k \in I_{n}}\left\{M\left(\frac{\bar{d}\left(\Delta_{p}^{q} X_{\sigma^{k}(m)}, X_{0}\right)}{\rho}\right)\right\}^{r_{k}} \geq \varepsilon\right\} \in I,
$$

and, consequently, $X_{k} \rightarrow X_{0} \in\left[V_{\sigma}, \lambda, \Delta_{p}^{q}, M, r\right]^{I(F)}$. 
Let $\lim _{k \rightarrow \infty} r_{k}=r>0$. Suppose that $X_{k} \rightarrow Y_{1} \in\left[V_{\sigma}, \lambda, \Delta_{p}^{q}, M, r\right]^{I(F)}, X_{k} \rightarrow Y_{2} \in\left[V_{\sigma}, \lambda, \Delta_{p}^{q}\right.$, $M, r]^{I(F)}$ and $\left(\bar{d}\left(Y_{1}, Y_{2}\right)\right)^{r_{k}}=a>0$.

Now, from (2) and the definition of Orlicz function, we have

$$
\begin{aligned}
\frac{1}{\lambda_{n}} \sum_{k \in I_{n}} M\left(\frac{\bar{d}\left(Y_{1}, Y_{2}\right)}{\rho}\right)^{r_{k}} \leq & \frac{C}{\lambda_{n}} \sum_{k \in I_{n}} M\left(\frac{\bar{d}\left(\Delta_{p}^{q} X_{\sigma^{k}(m)}, Y_{1}\right)}{\rho}\right)^{r_{k}} \\
& +\frac{C}{\lambda_{n}} \sum_{k \in I_{n}} M\left(\frac{\bar{d}\left(\Delta_{p}^{q} X_{\sigma^{k}(m)}, Y_{2}\right)}{\rho}\right)^{r_{k}} .
\end{aligned}
$$

Since

$$
\begin{aligned}
& \left\{n \in N: \lim _{n} \frac{1}{\lambda_{n}} \sum_{k \in I_{n}} M\left(\frac{\bar{d}\left(\Delta_{p}^{q} X_{\sigma^{k}(m)}, Y_{1}\right)}{\rho}\right)^{r_{k}} \geq \varepsilon\right\} \in I, \\
& \left\{n \in N: \lim _{n} \frac{1}{\lambda_{n}} \sum_{k \in I_{n}} M\left(\frac{\bar{d}\left(\Delta_{p}^{q} X_{\sigma^{k}(m)}, Y_{2}\right)}{\rho}\right)^{r_{k}} \geq \varepsilon\right\} \in I .
\end{aligned}
$$

Hence,

$$
\left\{n \in N: \lim _{n} \frac{1}{\lambda_{n}} \sum_{k \in I_{n}} M\left(\frac{\bar{d}\left(Y_{1}, Y_{2}\right)}{\rho}\right)^{r_{k}} \geq \varepsilon\right\} \in I .
$$

Further, $M\left(\frac{\bar{d}\left(Y_{1}, Y_{2}\right)}{\rho}\right)^{r} r_{k} \rightarrow M\left(\frac{a}{\rho}\right)^{r}$ as $k \rightarrow \infty$, and therefore,

$$
\lim _{n \rightarrow \infty} \frac{1}{\lambda_{n}} \sum_{k \in I_{n}} M\left(\frac{\bar{d}\left(Y_{1}, Y_{2}\right)}{\rho}\right)^{r_{k}}=M\left(\frac{a}{\rho}\right)^{r}
$$

From (3) and (4), it follows that $M\left(\frac{a}{\rho}\right)=0$, and by the definition of an Orlicz function, we have $a=0$.

Hence, $Y_{1}=Y_{2}$, and this completes the proof.

Theorem 3.3 (i) Let $0<\inf _{k} r_{k} \leq r_{k} \leq 1$. Then

$$
\left[V_{\sigma}, \lambda, \Delta_{p}^{q}, M, r\right]^{I(F)} \subset\left[V_{\sigma}, \lambda, \Delta_{p}^{q}, M\right]^{I(F)} .
$$

(ii) Let $0<r_{k} \leq \sup _{k} r_{k}<\infty$. Then

$$
\left[V_{\sigma}, \lambda, \Delta_{p}^{q}, M\right]^{I(F)} \subset\left[V_{\sigma}, \lambda, \Delta_{p}^{q}, M, r\right]^{I(F)} .
$$

Proof (i) Let $X \in\left[V_{\sigma}, \lambda, \Delta_{p}^{q}, M, r\right]^{I(F)}$. Since $0<\inf _{k} r_{k} \leq 1$, we get

$$
\frac{1}{\lambda_{n}} \sum_{k \in I_{n}} M\left(\frac{\bar{d}\left(\Delta_{p}^{q} X_{\sigma^{k}(m)}, X_{0}\right)}{\rho}\right) \leq \frac{1}{\lambda_{n}} \sum_{k \in I_{n}} M\left(\frac{\bar{d}\left(\Delta_{p}^{q} X_{\sigma^{k}(m)}, X_{0}\right)}{\rho}\right)^{r_{k}} .
$$


So,

$$
\begin{aligned}
& \left\{n \in N: \lim _{n} \frac{1}{\lambda_{n}} \sum_{k \in I_{n}}\left\{M\left(\frac{\bar{d}\left(\Delta_{p}^{q} X_{\sigma^{k}(m)}, X_{0}\right)}{\rho}\right)\right\} \geq \varepsilon, \text { uniformly in } m\right\} \\
& \subseteq\left\{n \in N: \lim _{n} \frac{1}{\lambda_{n}} \sum_{k \in I_{n}}\left\{M\left(\frac{\bar{d}\left(\Delta_{p}^{q} X_{\sigma^{k}(m)}, X_{0}\right.}{\rho}\right)\right\}^{r_{k}} \geq \varepsilon, \text { uniformly in } m\right\} \in I,
\end{aligned}
$$

and hence, $X \in\left[V_{\sigma}, \lambda, \Delta_{p}^{q}, M\right]^{I(F)}$.

(ii) Let $r \geq 1$ and $\sup _{k} r_{k}<\infty$. Let $X \in\left[V_{\sigma}, \lambda, \Delta_{p}^{q}, M\right]^{I(F)}$. Then for each $k, 0<\varepsilon<1$, there exists a positive integer $N$ such that

$$
\frac{1}{\lambda_{n}} \sum_{k \in I_{n}} M\left(\frac{\bar{d}\left(\Delta_{p}^{q} X_{\sigma^{k}(m)}, X_{0}\right)}{\rho}\right) \leq \varepsilon<1
$$

for all $m \geq N$. This implies that

$$
\frac{1}{\lambda_{n}} \sum_{k \in I_{n}} M\left(\frac{\bar{d}\left(\Delta_{p}^{q} X_{\sigma^{k}(m)}, X_{0}\right)}{\rho}\right)^{r_{k}} \leq \frac{1}{\lambda_{n}} \sum_{k \in I_{n}} M\left(\frac{\bar{d}\left(\Delta_{p}^{q} X_{\sigma^{k}(m)}, X_{0}\right)}{\rho}\right) .
$$

So,

$$
\begin{aligned}
& \left\{n \in N: \frac{1}{\lambda_{n}} \sum_{k \in I_{n}}\left\{M\left(\frac{\bar{d}\left(\Delta_{p}^{q} X_{\sigma^{k}(m)}, X_{0}\right)}{\rho}\right)\right\}^{r_{k}} \geq \varepsilon, \text { uniformly in } m\right\} \\
& \subseteq\left\{n \in N: \frac{1}{\lambda_{n}} \sum_{k \in I_{n}}\left\{M\left(\frac{\bar{d}\left(\Delta_{p}^{q} X_{\sigma^{k}(m)}, X_{0}\right)}{\rho}\right)\right\} \geq \varepsilon \text {, uniformly in } m\right\} \in I .
\end{aligned}
$$

Therefore, $X \in\left[V_{\sigma}, \lambda, \Delta_{p}^{q}, M, r\right]^{I(F)}$.

This completes the proof.

Theorem 3.4 Let $X^{F}\left(V_{\sigma}, \lambda, \Delta_{p}^{q-1}\right)$ stand for $\left[V_{\sigma}, \lambda, \Delta_{p}^{q-1}, M, r\right]_{0}^{I(F)},\left[V_{\sigma}, \lambda, \Delta_{p}^{q-1}, M, r\right]^{I(F)}$ or $\left[V_{\sigma}, \lambda, \Delta_{p}^{q-1}, M, r\right]_{\infty}^{I(F)}$ and $m \geq 1$. Then the inclusion $X^{F}\left(V_{\sigma}, \lambda, \Delta_{p}^{q-1}\right) \subset X^{F}\left(V_{\sigma}, \lambda, \Delta_{p}^{q}\right)$ is strict. In general, $X^{F}\left(V_{\sigma}, \lambda, \Delta_{p}^{i}\right) \subset X\left(V_{\sigma}, \lambda, \Delta_{p}^{q}\right)$ for all $i=1,2,3, \ldots, p-1$ and the inclusion is strict.

Proof Let us take $\left[V_{\sigma}, \lambda, \Delta_{p}^{q-1}, M, r\right]_{0}^{I(F)}$.

Let $X=\left(X_{k}\right) \in\left[V_{\sigma}, \lambda, \Delta_{p}^{q-1}, M, r\right]_{0}^{I(F)}$. Then for given $\varepsilon>0$, we have

$$
\left\{n \in N: \frac{1}{\lambda_{n}} \sum_{k \in I_{n}}\left\{M\left(\frac{\bar{d}\left(\Delta_{p}^{q-1} X_{\sigma^{k}(m)}, \overline{0}\right)}{\rho}\right)\right\}^{r_{k}} \geq \varepsilon\right\} \in I \quad \text { for some } \rho>0 .
$$

Since $M$ is non-decreasing and convex, it follows that

$$
\begin{aligned}
& \frac{1}{\lambda_{n}} \sum_{k \in I_{n}}\left\{M\left(\frac{\bar{d}\left(\Delta_{p}^{q} X_{\sigma^{k}(m)}, \overline{0}\right)}{\rho}\right)\right\}^{r_{k}} \\
& \quad=\frac{1}{\lambda_{n}} \sum_{k \in I_{n}}\left\{M\left(\frac{\bar{d}\left(\Delta_{p}^{q-1} X_{\sigma^{k+1}(m)}, \Delta_{p}^{q-1} X_{\sigma^{k}(m)}\right)}{\rho}\right)\right\}^{r_{k}}
\end{aligned}
$$




$$
\begin{aligned}
& \leq D \frac{1}{\lambda_{n}} \sum_{k \in I_{n}}\left(\left[\frac{1}{2} M\left(\frac{\bar{d}\left(\Delta_{p}^{q-1} X_{\sigma^{k+1}(m)}, \overline{0}\right)}{\rho}\right)\right]^{r_{k}}+\left[\frac{1}{2} M\left(\frac{\bar{d}\left(\Delta_{p}^{q-1} X_{\sigma^{k}(m)}, \overline{0}\right)}{\rho}\right)\right]^{r_{k}}\right) \\
& \leq D \frac{1}{\lambda_{n}} \sum_{k \in I_{n}}\left(\left[M\left(\frac{\bar{d}\left(\Delta_{p}^{q-1} X_{\sigma^{k+1}(m)}, \overline{0}\right)}{\rho}\right)\right]^{r_{k}}+\left[M\left(\frac{\bar{d}\left(\Delta_{p}^{q-1} X_{\sigma^{k}(m)}, \overline{0}\right)}{\rho}\right)\right]^{r_{k}}\right) .
\end{aligned}
$$

Hence, we have

$$
\begin{aligned}
& \left\{n \in N: \frac{1}{\lambda_{n}} \sum_{k \in I_{n}}\left\{M\left(\frac{\bar{d}\left(\Delta_{p}^{q} X_{\sigma^{k}(m)}, \overline{0}\right)}{\rho}\right)\right\}^{r_{k}} \geq \varepsilon\right\} \\
& \subseteq\left\{n \in N: D \frac{1}{\lambda_{n}} \sum_{k \in I_{n}}\left\{M\left(\frac{\bar{d}\left(\Delta_{p}^{q-1} X_{\sigma^{k+1}(m)}, \overline{0}\right)}{\rho}\right)\right\}^{r_{k}} \geq \frac{\varepsilon}{2}\right\} \\
& \cup\left\{n \in N: D \frac{1}{\lambda_{n}} \sum_{k \in I_{n}}\left\{M\left(\frac{\bar{d}\left(\Delta_{p}^{q-1} X_{\sigma^{k}(m)}, \overline{0}\right)}{\rho}\right)\right\}^{r_{k}} \geq \frac{\varepsilon}{2}\right\} .
\end{aligned}
$$

Since the set on the right-hand side belongs to $I$, so does the left-hand side. The inclusion is strict as the sequence $X=\left(k^{r}\right)$, for example, belongs to $\left[V_{\sigma}, \lambda, \Delta_{p}^{q}, M\right]_{0}^{I(F)}$ but does not belong to $\left[V_{\sigma}, \lambda, \Delta_{p}^{q-1}, M\right]_{0}^{I(F)}$ for $M(x)=x$ and $r_{k}=1$ for all $k$.

Theorem $3.5\left[V_{\sigma}, \lambda, \Delta_{p}^{q}, M, r\right]_{0}^{I(F)}$ and $\left[V_{\sigma}, \lambda, \Delta_{p}^{q}, M, r\right]^{I(F)}$ are complete metric spaces, with the metric defined by

$$
\begin{aligned}
\overline{d_{\sigma}}(X, Y)= & \sum_{m=1}^{p q} \bar{d}\left(X_{\sigma^{k}(m)}, Y_{\sigma^{k}(m)}\right) \\
& +\inf \left\{\rho^{\frac{r_{k}}{H}}: \sup _{m, n}\left(\frac{1}{\lambda_{n}} \sum_{k \in I_{n}}\left(M\left(\frac{\bar{d}\left(\Delta_{p}^{q} X_{\sigma^{k}(m)}, \Delta_{p}^{q} Y_{\sigma^{k}(m)}\right)}{\rho}\right)\right)^{H}\right) \leq 1\right. \\
& \text { for some } \rho>0\},
\end{aligned}
$$

where $H=\max \left(1,\left(\sup _{k} r_{k}\right)\right)$.

\section{Competing interests}

The authors declare that they have no competing interests.

\section{Authors' contributions}

Both of the authors contributed equally. The authors also read the galley proof and approved the final copy of the manuscript.

\section{Author details}

'Department of Mathematics and Institute for Mathematical Research, University of Putra Malaysia, Serdang, Selangor 43400, Malaysia. ${ }^{2}$ Department of Mathematics, Indian Institute of Technology, Bombay Powai: 400076, Mumbai, Maharashtra, India.

\section{Acknowledgements}

The authors are very grateful to the referees for the very useful comments and for detailed remarks that improved the presentation and the contents of the manuscript. The work of the authors was carried under the Post Doctoral Fellow under National Board of Higher Mathematics, DAE, project No. NBHM/PDF.50/2011/64. The first author gratefully acknowledges that the present work was partially supported under the Post Doctoral Fellow under National Board of Higher Mathematics, DAE, project No. NBHM/PDF.50/2011/64. The second author also acknowledges that the part of the work was supported by the University Putra Malaysia, Grant ERGS 5527179. 


\section{References}

1. Lorentz, GG: A contribution to the theory of divergent sequences. Acta Math. 80, 167-190 (1948)

2. Maddox, IJ: Spaces of strongly summable sequences. Q. J. Math. 18, 345-355 (1967)

3. Schaefer, P: Infinite matrices and invariant means. Proc. Am. Math. Soc. 36, 104-110 (1972)

4. Mursaleen, M: Matrix transformations between some new sequence spaces. Houst. J. Math. 9(4), 505-509 (1993)

5. Kostyrko, P, Šalăt, T, Wilczyński, W: On I-convergence. Real Anal. Exch. 26(2), 669-685 (2000-2001)

6. Lindenstrauss, J, Tzafriri, L: On Orlicz sequence spaces. Isr. J. Math. 10, 379-390 (1971)

7. Kizmaz, H: On certain sequence spaces. Can. Math. Bull. 24(2), 169-176 (1981)

8. Leindler, L: Über die verallgemeinerte de la Vallée-Poussinsche summierbarkeit allgemeiner Orthogonalreihen. Acta Math. Acad. Sci. Hung. 16, 375-387 (1965)

9. Nuray, F, Savaş, E: On strong almost A-summability with respect to a modulus and statistical convergence. Indian J. Pure Appl. Math. 23(3), 217-222 (1992)

10. Savaş, E, Kılıçman, A: A note on some strongly sequence spaces. Abstr. Appl. Anal. 2011, Article ID 598393 (2011)

10.1186/1687-1847-2013-288

Cite this article as: Kilıçman and Borgohain: Generalized difference strongly summable sequence spaces of fuzzy real numbers defined by ideal convergence and Orlicz function. Advances in Difference Equations 2013, 2013:288

\section{Submit your manuscript to a SpringerOpen ${ }^{\circ}$ journal and benefit from:}

- Convenient online submission

Rigorous peer review

- Immediate publication on acceptance

- Open access: articles freely available online

- High visibility within the field

- Retaining the copyright to your article 